International Mathematical Forum, 1, 2006, no. 39, 1905-1912

\title{
On an Extended Hardy-Hilbert's Inequality and Some Reversed Form
}

\author{
Bicheng Yang \\ Department of Mathematics \\ Guangdong Education Institute \\ Guangzhou 510303, P. R. China \\ bcyang@pub.guangzhou.gd.cn
}

\begin{abstract}
By introducing a parameter $\lambda$ and the $\beta$ function, we give a new extended Hardy-Hilbert's inequality with a best constant factor and the equivalent form. Some reversed form for any particular parameter are considered.
\end{abstract}

Keywords: Hardy-Hilbert's inequality; Weight coefficient; Hölder's inequality

Mathematics Subject Classification: 26D15

\section{Introduction}

If $p>1, \frac{1}{p}+\frac{1}{q}=1, a_{n}, b_{n} \geq 0(n \in N)$, such that $0<\sum_{n=1}^{\infty} a_{n}^{p}<\infty$ and $0<\sum_{n=1}^{\infty} b_{n}^{q}<\infty$, then the well known Hardy- Hilbert's inequality and the equivalent form are as follows ( see Hardy et al. [1] ):

$$
\begin{aligned}
& \sum_{n=1}^{\infty} \sum_{m=1}^{\infty} \frac{a_{m} b_{n}}{m+n}<\frac{\pi}{\sin (\pi / p)}\left\{\sum_{n=1}^{\infty} a_{n}^{p}\right\}^{1 / p}\left\{\sum_{n=1}^{\infty} b_{n}^{q}\right\}^{1 / q} ; \\
& \sum_{n=1}^{\infty}\left(\sum_{m=1}^{\infty} \frac{a_{m}}{m+n}\right)^{p}<\left[\frac{\pi}{\sin (\pi / p)}\right]^{p} \sum_{n=1}^{\infty} a_{n}^{p},
\end{aligned}
$$

where the constant factors $\pi / \sin (\pi / p)$ and $[\pi / \sin (\pi / p)]^{p}$ are the best possible. Inequality (1.1) is important in analysis and its applications ( see Mitinovic [2] ). In recent years, (1.1) had been strengthened by [3, 4]. By introducing a parameter, Yang $[5,6]$ gave two extensions of (1.1) as: If the series in the right of the following inequalities converge to some positive numbers, then

$$
\sum_{n=1}^{\infty} \sum_{m=1}^{\infty} \frac{a_{m} b_{n}}{(m+n)^{\lambda}}<B\left(\frac{p+\lambda-2}{p}, \frac{q+\lambda-2}{q}\right)\left\{\sum_{n=1}^{\infty} n^{1-\lambda} a_{n}^{p}\right\}^{\frac{1}{p}}\left\{\sum_{n=1}^{\infty} n^{1-\lambda} b_{n}^{q}\right\}^{\frac{1}{q}},
$$


where the constant factor $B\left(\frac{p+\lambda-2}{p}, \frac{q+\lambda-2}{q}\right)(2-\min \{p, q\}<\lambda \leq 2)$ is the best possible ( $\mathrm{B}(\mathrm{u}, \mathrm{v})$ is the $\beta$ function); and the other is

$$
\sum_{n=1}^{\infty} \sum_{m=1}^{\infty} \frac{a_{m} b_{n}}{m^{\lambda}+n^{\lambda}}<\frac{\pi}{\lambda \sin \left(\frac{\pi}{p}\right)}\left\{\sum_{n=1}^{\infty} n^{(p-1)(1-\lambda)} a_{n}^{p}\right\}^{\frac{1}{p}}\left\{\sum_{n=1}^{\infty} n^{(q-1)(1-\lambda)} b_{n}^{q}\right\}^{\frac{1}{q}},
$$

where the constant factor $\pi /[\lambda \sin (\pi / p)](0<\lambda \leq \min \{p, q\})$ is the best possible. For $\lambda=1$, both (1.3) and (1.4) reduce to (1.1). In 2003, Yang et al. [7] summarized the way of weight coefficient on research for Hilbert-type inequalities. More recently, Zhao [8] considered some inverses of Pachpatte's inequalities.

The main objective of this paper is to build a new extended form of (1.1) with a best constant factor, related the left double series of (1.4). As applications, an equivalent form and some reversed form for $\lambda=2$ are considered.

\section{Some Lemmas and Main Results}

We need the formula of the $\beta$ function $B(p, q)$ as follows ( see [9]):

$$
B(p, q)=B(q, p)=\int_{0}^{\infty} \frac{u^{p-1}}{(1+u)^{p+q}} d u(p, q>0) .
$$

Lemma 2.1 If $r>1, \frac{1}{r}+\frac{1}{s}=1,2-\min \{r, s\}<\lambda \leq 2$, define $\varpi_{\lambda}(n, r)$ as

$$
\varpi_{\lambda}(n, r):=n^{\lambda-1} \sum_{m=1}^{\infty} \frac{1}{m^{\lambda}+n^{\lambda}}\left(\frac{n}{m}\right)^{\frac{2-\lambda}{r}}(n \in N) .
$$

Then one has

$$
\varpi_{\lambda}(n, r)<\frac{1}{\lambda} B\left(\frac{r+\lambda-2}{r \lambda}, \frac{s+\lambda-2}{s \lambda}\right) .
$$

Proof Since $0 \leq 2-\min \{r, s\}<\lambda \leq 2$, by (2.2), we have

$$
\varpi_{\lambda}(n, r)<n^{\lambda-1} \int_{0}^{\infty} \frac{1}{x^{\lambda}+n^{\lambda}}\left(\frac{n}{x}\right)^{\frac{2-\lambda}{r}} d x .
$$

Setting $t=(x / n)^{\lambda}$ in the above integral, we find

$$
\varpi_{\lambda}(n, r)<\frac{1}{\lambda} \int_{0}^{\infty} \frac{1}{t+1} t^{\frac{r+\lambda-2}{r \lambda}-1} d t .
$$

Then by (2.1), since $r+\lambda-2>0, s+\lambda-2>0$ and $\frac{r+\lambda-2}{r \lambda}+\frac{s+\lambda-2}{s \lambda}=1$, we have (2.3). The lemma is proved.

Lemma 2.2 If $p>1, \frac{1}{p}+\frac{1}{q}=1,2-\min \{p, q\}<\lambda \leq 2$, and $0<\varepsilon<$ $\frac{q+\lambda-2}{2}$, then

$$
I:=\sum_{n=1}^{\infty} \sum_{m=1}^{\infty} \frac{1}{m^{\lambda}+n^{\lambda}} m^{\frac{\lambda-2-\varepsilon}{p}} n^{\frac{\lambda-2-\varepsilon}{q}}
$$




$$
>\frac{1}{\varepsilon \lambda} B\left(\frac{q+\lambda-2}{q \lambda}-\frac{\varepsilon}{q \lambda}, \frac{p+\lambda-2}{p \lambda}+\frac{\varepsilon}{q \lambda}\right)-\left(\frac{2 q}{q+\lambda-2}\right) .
$$

Proof We have

$$
I>\int_{1}^{\infty} x^{\frac{\lambda-2-\varepsilon}{p}}\left[\int_{1}^{\infty} \frac{1}{x^{\lambda}+y^{\lambda}} y^{\frac{\lambda-2-\varepsilon}{q}} d y\right] d x .
$$

Setting $u=(y / x)^{\lambda}$ in the above integral, we obtain

$$
\begin{aligned}
& I>\frac{1}{\lambda} \int_{1}^{\infty} x^{-1-\varepsilon}\left[\int_{1 / x^{\lambda}}^{\infty} \frac{1}{1+u} u^{\frac{q+\lambda-2-\varepsilon}{\lambda q}-1} d u\right] d x \\
& =\frac{1}{\lambda} \int_{1}^{\infty} x^{-1-\varepsilon}\left[\int_{0}^{\infty} \frac{1}{1+u} u^{\frac{q+\lambda-2-\varepsilon}{\lambda q}-1} d u\right] d x \\
& \quad-\frac{1}{\lambda} \int_{1}^{\infty} x^{-1-\varepsilon}\left[\int_{0}^{\frac{1}{x^{\lambda}}} \frac{1}{1+u} u^{\frac{q+\lambda-2-\varepsilon}{\lambda q}-1} d u\right] d x \\
& =\frac{1}{\varepsilon \lambda} B\left(\frac{q+\lambda-2}{q \lambda}-\frac{\varepsilon}{\lambda q}, \frac{p+\lambda-2}{p \lambda}+\frac{\varepsilon}{\lambda q}\right) \\
& \quad-\frac{1}{\lambda} \int_{1}^{\infty} x^{-1-\varepsilon}\left[\int_{0}^{\frac{1}{x^{\lambda}}} \frac{1}{1+u} u^{\frac{q+\lambda-2-\varepsilon}{\lambda q}-1} d u\right] d x .
\end{aligned}
$$

Since $0 \leq 2-\min \{p, q\}<\lambda \leq 2$, and $0<\varepsilon<(q+\lambda-2) / 2$, we find

$$
\begin{aligned}
& \frac{1}{\lambda} \int_{1}^{\infty} x^{-1-\varepsilon}\left[\int_{0}^{\frac{1}{x^{\lambda}}} \frac{1}{1+u} u^{\frac{q+\lambda-2-\varepsilon}{\lambda q}-1} d u\right] d x \\
< & \frac{1}{\lambda} \int_{1}^{\infty} x^{-1}\left[\int_{0}^{\frac{1}{x^{\lambda}}} u^{\frac{q+\lambda-2}{2 \lambda q}-1} d u\right] d x=\left(\frac{2 q}{q+\lambda-2}\right),
\end{aligned}
$$

then by (2.5), we have (2.4). The lemma is proved.

Theorem 2.3 If $p>1, \frac{1}{p}+\frac{1}{q}=1,2-\min \{p, q\}<\lambda \leq 2, a_{n}, b_{n} \geq 0$, such that $0<\sum_{n=1}^{\infty} n^{1-\lambda} a_{n}^{p}<\infty$ and $0<\sum_{n=1}^{\infty} n^{1-\lambda} b_{n}^{q}<\infty$, then we have

$$
\sum_{n=1}^{\infty} \sum_{m=1}^{\infty} \frac{a_{m} b_{n}}{m^{\lambda}+n^{\lambda}}<\frac{1}{\lambda} B\left(\frac{p+\lambda-2}{p \lambda}, \frac{q+\lambda-2}{q \lambda}\right)\left\{\sum_{n=1}^{\infty} n^{1-\lambda} a_{n}^{p}\right\}^{\frac{1}{p}}\left\{\sum_{n=1}^{\infty} n^{1-\lambda} b_{n}^{q}\right\}^{\frac{1}{q}},
$$

where the constant factor $\frac{1}{\lambda} B\left(\frac{p+\lambda-2}{p \lambda}, \frac{q+\lambda-2}{q \lambda}\right)$ is the best possible.

Proof By Hölder's inequality and (2.2), one has

$$
\begin{gathered}
\sum_{n=1}^{\infty} \sum_{m=1}^{\infty} \frac{a_{m} b_{n}}{m^{\lambda}+n^{\lambda}}=\sum_{n=1}^{\infty} \sum_{m=1}^{\infty}\left[\frac{a_{m}}{\left(m^{\lambda}+n^{\lambda}\right)^{\frac{1}{p}}}\left(\frac{m}{n}\right)^{\frac{2-\lambda}{p q}}\right]\left[\frac{b_{n}}{\left(m^{\lambda}+n^{\lambda}\right)^{\frac{1}{q}}}\left(\frac{n}{m}\right)^{\frac{2-\lambda}{p q}}\right] \\
\leq\left\{\sum_{m=1}^{\infty} \sum_{n=1}^{\infty} \frac{a_{m}^{p}}{m^{\lambda}+n^{\lambda}}\left(\frac{m}{n}\right)^{\frac{2-\lambda}{q}}\right\}^{\frac{1}{p}}\left\{\sum_{n=1}^{\infty} \sum_{m=1}^{\infty} \frac{b_{n}^{q}}{m^{\lambda}+n^{\lambda}}\left(\frac{n}{m}\right)^{\frac{2-\lambda}{p}}\right\}^{\frac{1}{q}}
\end{gathered}
$$




$$
=\left\{\sum_{m=1}^{\infty} \varpi_{\lambda}(m, q) m^{1-\lambda} a_{m}^{p}\right\}^{\frac{1}{p}}\left\{\sum_{n=1}^{\infty} \varpi_{\lambda}(n, p) n^{1-\lambda} b_{n}^{q}\right\}^{\frac{1}{q}} .
$$

Hence by (2.3) and (2.1), we have (2.6).

For $0<\varepsilon<(q+\lambda-2) / 2$, setting $\tilde{a}_{n}, \tilde{b}_{n}$ as: $\tilde{a}_{n}=n^{(\lambda-2-\varepsilon) / p} ; \tilde{b}_{n}=$ $n^{(\lambda-2-\varepsilon) / q}, n \in N$, then we find

$$
\begin{aligned}
& \left\{\sum_{n=1}^{\infty} n^{1-\lambda} \tilde{a}_{n}^{p}\right\}^{\frac{1}{p}}\left\{\sum_{n=1}^{\infty} n^{1-\lambda} \tilde{b}_{n}^{q}\right\}^{\frac{1}{q}}=1+\sum_{n=2}^{\infty} n^{-1-\varepsilon} \\
& <1+\int_{1}^{\infty} x^{-1-\varepsilon} d x=1+\frac{1}{\varepsilon} .
\end{aligned}
$$

If there exists a parameter $\lambda$, such that the constant factor $\frac{1}{\lambda} B\left(\frac{p+\lambda-2}{p \lambda}, \frac{q+\lambda-2}{q \lambda}\right)$ in (2.6) is not the best possible, then there exists a positive number $\mathrm{k}$ with $k<$ $\frac{1}{\lambda} B\left(\frac{p+\lambda-2}{\lambda}, \frac{q+\lambda-2}{q \lambda}\right)$, such that $(2.6)$ is still value if one replaces $\frac{1}{\lambda} B\left(\frac{p+\lambda-2}{p \lambda}, \frac{q+\lambda-2}{q \lambda}\right)$ by $\mathrm{k}$. In particular, one has

$$
\varepsilon \sum_{n=1}^{\infty} \sum_{m=1}^{\infty} \frac{\tilde{a}_{m} \tilde{b}_{n}}{m^{\lambda}+n^{\lambda}}<\varepsilon k\left\{\sum_{n=1}^{\infty} n^{1-\lambda} \tilde{a}_{n}^{p}\right\}^{1 / p}\left\{\sum_{n=1}^{\infty} n^{1-\lambda} \tilde{b}_{n}^{q}\right\}^{1 / q} .
$$

Hence by (2.4) and (2.7), we obtain

$$
\frac{1}{\lambda} B\left(\frac{q+\lambda-2}{q \lambda}-\frac{\varepsilon}{\lambda q}, \frac{p+\lambda-2}{p \lambda}+\frac{\varepsilon}{\lambda q}\right)-\varepsilon\left(\frac{2 q}{q+\lambda-2}\right)<k(1+\varepsilon),
$$

and $\frac{1}{\lambda} B\left(\frac{p+\lambda-2}{p \lambda}, \frac{q+\lambda-2}{q \lambda}\right) \leq k\left(\varepsilon \rightarrow 0^{+}\right)$. This contradicts that $k<\frac{1}{\lambda} B\left(\frac{p+\lambda-2}{p \lambda}, \frac{q+\lambda-2}{q \lambda}\right)$. Hence the constant factor $\frac{1}{\lambda} B\left(\frac{p+\lambda-2}{p \lambda}, \frac{q+\lambda-2}{q \lambda}\right)$ in (2.6) is the best possible. The theorem is proved.

Theorem 2.4 If $p>1, \frac{1}{p}+\frac{1}{q}=1,2-\min \{p, q\}<\lambda \leq 2, a_{n} \geq 0$, such that $0<\sum_{n=1}^{\infty} n^{1-\lambda} a_{n}^{p}<\infty$, then

$$
\sum_{n=1}^{\infty} n^{(p-1)(\lambda-1)}\left(\sum_{m=1}^{\infty} \frac{a_{m}}{m^{\lambda}+n^{\lambda}}\right)^{p}<\left[\frac{1}{\lambda} B\left(\frac{p+\lambda-2}{p \lambda}, \frac{q+\lambda-2}{q \lambda}\right)\right]^{p} \sum_{n=1}^{\infty} n^{1-\lambda} a_{n}^{p},
$$

where the constant factor $\left[\frac{1}{\lambda} B\left(\frac{p+\lambda-2}{p \lambda}, \frac{q+\lambda-2}{q \lambda}\right)\right]^{p}$ is the best possible; Inequality (2.8) is equivalent to (2.6). In particular, for $\lambda=2$, one has

$$
\sum_{n=1}^{\infty} n^{p-1}\left(\sum_{m=1}^{\infty} \frac{a_{m}}{m^{2}+n^{2}}\right)^{p}<\left(\frac{\pi}{2}\right)^{p} \sum_{n=1}^{\infty} \frac{a_{n}^{p}}{n} .
$$

Proof Setting $b_{n}$ as: $b_{n}=n^{(p-1)(\lambda-1)}\left(\sum_{m=1}^{\infty} \frac{a_{m}}{m^{\lambda}+n^{\lambda}}\right)^{p-1}$, then by (2.6), we find

$$
0<\sum_{n=1}^{\infty} n^{1-\lambda} b_{n}^{q}=\sum_{n=1}^{\infty} n^{(p-1)(\lambda-1)}\left(\sum_{m=1}^{\infty} \frac{a_{m}}{m^{\lambda}+n^{\lambda}}\right)^{p}=\sum_{n=1}^{\infty} \sum_{m=1}^{\infty} \frac{a_{m} b_{n}}{m^{\lambda}+n^{\lambda}}
$$




$$
\begin{gathered}
\leq \frac{1}{\lambda} B\left(\frac{p+\lambda-2}{p \lambda}, \frac{q+\lambda-2}{q \lambda}\right)\left\{\sum_{n=1}^{\infty} n^{1-\lambda} a_{n}^{p}\right\}^{\frac{1}{p}}\left\{\sum_{n=1}^{\infty} n^{1-\lambda} b_{n}^{q}\right\}^{\frac{1}{q}} \\
0<\sum_{n=1}^{\infty} n^{1-\lambda} b_{n}^{q} \leq\left[\frac{1}{\lambda} B\left(\frac{p+\lambda-2}{p \lambda}, \frac{q+\lambda-2}{q \lambda}\right)\right]^{p} \sum_{n=1}^{\infty} n^{1-\lambda} a_{n}^{p}<\infty .
\end{gathered}
$$

Hence (2.10) takes the form of strict inequality using (2.6); so is (2.11). Which shows (2.8).

If (2.8) is valid, then by Hölder's inequality, we have

$$
\begin{aligned}
& \sum_{n=1}^{\infty} \sum_{m=1}^{\infty} \frac{a_{m} b_{n}}{m^{\lambda}+n^{\lambda}}=\sum_{n=1}^{\infty}\left[n^{(\lambda-1) / q} \sum_{m=1}^{\infty} \frac{a_{m}}{m^{\lambda}+n^{\lambda}}\right]\left[n^{(1-\lambda) / q} b_{n}\right] \\
\leq & \left\{\sum_{n=1}^{\infty} n^{(p-1)(\lambda-1)}\left(\sum_{m=1}^{\infty} \frac{a_{m}}{m^{\lambda}+n^{\lambda}}\right)^{p}\right\}^{\frac{1}{p}}\left\{\sum_{n=1}^{\infty} n^{1-\lambda} b_{n}^{q}\right\}^{\frac{1}{q}} .
\end{aligned}
$$

Hence by (2.8), we have (2.6). It follows that (2.8) is equivalent to (2.6).

If the constant factor in (2.8) is not the best possible, we can get a contradiction from (2.12) that the constant factor in (2.6) is not the best possible. The theorem is proved.

Remark 2.5 For $\lambda=1$, (2.6) and (2.8) reduce respectively to (1.1) and (1.2). It follows that (2.6) is a generalization of (1.1), and (2.8) is a generalization of (1.2). Although inequalities (2.6), (1.3) and (1.4) are with respectively a parameter $\lambda$, but they are different.

\section{Some Reversed Inequalities for $\lambda=2$}

Lemma 3.1 If $0<p<1, \frac{1}{p}+\frac{1}{q}=1$ and $0<\varepsilon<p$, then

$$
J:=\sum_{n=1}^{\infty} \sum_{m=1}^{\infty} \frac{m^{-\varepsilon / p} n^{-\varepsilon / q}}{m^{2}+n^{2}}<\frac{1}{2} B\left(\frac{p-\varepsilon}{2 p}, \frac{p+\varepsilon}{2 p}\right) \sum_{n=1}^{\infty} \frac{1}{n^{1+\varepsilon}} .
$$

Proof Since $0<p<1$, we find

$$
\sum_{m=1}^{\infty} \frac{m^{-\varepsilon / p} n^{-\varepsilon / q}}{m^{2}+n^{2}}<\int_{0}^{\infty} \frac{x^{-\varepsilon / p} n^{-\varepsilon / q}}{x^{2}+n^{2}} d x .
$$

Setting $y=(x / n)^{2}$ in the above integral, we obtain $d x=\frac{n}{2} y^{-1 / 2} d y$ and

$$
J<\sum_{n=1}^{\infty} \int_{0}^{\infty} \frac{x^{-\varepsilon / p} n^{-\varepsilon / q}}{x^{2}+n^{2}} d x=\frac{1}{2} \int_{0}^{\infty} \frac{1}{1+y} y^{\frac{p-\varepsilon}{2 p}-1} d y \sum_{n=1}^{\infty} \frac{1}{n^{1+\varepsilon}} .
$$

Hence by (2.1), we have (3.1). The lemma is proved. 
Theorem 3.2 If $0<p<1, \frac{1}{p}+\frac{1}{q}=1, a_{n}, b_{n} \geq 0$, such that $0<$ $\sum_{n=1}^{\infty} \frac{a_{n}^{p}}{n}<\infty$ and $0<\sum_{n=1}^{\infty} \frac{b_{n}^{q}}{n}<\infty$, then we have

$$
\sum_{n=1}^{\infty} \sum_{m=1}^{\infty} \frac{a_{m} b_{n}}{m^{2}+n^{2}}>\frac{\pi}{2}\left\{\sum_{n=1}^{\infty}\left(1-\frac{2}{\pi n}\right) \frac{a_{n}^{p}}{n}\right\}^{\frac{1}{p}}\left\{\sum_{n=1}^{\infty} \frac{b_{n}^{q}}{n}\right\}^{\frac{1}{q}}
$$

where the constant factor $\frac{\pi}{2}$ is the best possible.

Proof By the reverse of Hölder's inequality, one has

$$
\begin{aligned}
& \sum_{n=1}^{\infty} \sum_{m=1}^{\infty} \frac{a_{m} b_{n}}{m^{2}+n^{2}}=\sum_{n=1}^{\infty} \sum_{m=1}^{\infty}\left[\frac{a_{m}}{\left(m^{2}+n^{2}\right)^{\frac{1}{p}}}\right]\left[\frac{b_{n}}{\left(m^{2}+n^{2}\right)^{\frac{1}{q}}}\right] \\
\geq & \left\{\sum_{m=1}^{\infty} \sum_{n=1}^{\infty} \frac{a_{m}^{p}}{m^{2}+n^{2}}\right\}^{1 / p}\left\{\sum_{m=1}^{\infty} \sum_{n=1}^{\infty} \frac{b_{n}^{q}}{m^{2}+n^{2}}\right\}^{1 / q} \\
= & \left\{\sum_{m=1}^{\infty}\left(\sum_{n=1}^{\infty} \frac{m}{m^{2}+n^{2}}\right) \frac{a_{m}^{p}}{m}\right\}^{1 / p}\left\{\sum_{n=1}^{\infty}\left(\sum_{m=1}^{\infty} \frac{n}{m^{2}+n^{2}}\right) \frac{b_{n}^{q}}{n}\right\}^{1 / q} \\
= & \left\{\sum_{m=1}^{\infty} \omega(m) \frac{a_{m}^{p}}{m}\right\}^{1 / p}\left\{\sum_{n=1}^{\infty} \omega(n) \frac{b_{n}^{q}}{n}\right\}^{1 / q},
\end{aligned}
$$

where, the weight coefficient $\omega(j)$ is defined by $\omega(j):=\sum_{k=1}^{\infty} \frac{j}{j^{2}+k^{2}}(j=m, n)$.

Since we find

$$
\begin{gathered}
\omega(n)<\int_{0}^{\infty} \frac{n}{n^{2}+x^{2}} d x=\left[\arctan \frac{x}{n}\right]_{0}^{\infty}=\frac{\pi}{2} \\
\omega(m)>\int_{1}^{\infty} \frac{m}{m^{2}+x^{2}} d x=\frac{\pi}{2}-\arctan \frac{1}{m}>\frac{\pi}{2}-\frac{1}{m},
\end{gathered}
$$

then in view of $0<p<1, q<0$ and (3.3), we have (3.2).

For $0<\varepsilon<p$, setting $\tilde{a}_{n}, \tilde{b}_{n}$ as: $\tilde{a}_{n}=n^{-\varepsilon / p} ; \tilde{b}_{n}=n^{-\varepsilon / q}$, then we find

$$
\begin{gathered}
\left\{\sum_{n=1}^{\infty}\left(1-\frac{2}{\pi n}\right) \frac{\tilde{a}_{n}^{p}}{n}\right\}^{\frac{1}{p}}\left\{\sum_{n=1}^{\infty} \frac{\tilde{b}_{n}^{q}}{n}\right\}^{\frac{1}{q}}=\left\{\sum_{n=1}^{\infty}\left(1-\frac{2}{\pi n}\right) \frac{1}{n^{1+\varepsilon}}\right\}^{\frac{1}{p}}\left\{\sum_{n=1}^{\infty} \frac{1}{n^{1+\varepsilon}}\right\}^{\frac{1}{q}} \\
>\left\{\sum_{n=1}^{\infty} \frac{1}{n^{1+\varepsilon}}-\frac{2}{\pi} \sum_{n=1}^{\infty} \frac{1}{n^{2}}\right\}^{\frac{1}{p}}\left\{\sum_{n=1}^{\infty} \frac{1}{n^{1+\varepsilon}}\right\}^{\frac{1}{q}}=(1-o(1))^{\frac{1}{p}} \sum_{n=1}^{\infty} \frac{1}{n^{1+\varepsilon}}\left(\varepsilon \rightarrow 0^{+}\right) .
\end{gathered}
$$

If the constant factor $\frac{\pi}{2}$ in (3.2) is not the best possible, then there exists a positive number $k>\frac{\pi}{2}$, such that (3.2) is still valid if one replaces $\frac{\pi}{2}$ by $\mathrm{k}$. In particular, one has

$$
\sum_{n=1}^{\infty} \sum_{m=1}^{\infty} \frac{\tilde{a}_{m} \tilde{b}_{n}}{m^{2}+n^{2}}>k\left\{\sum_{n=1}^{\infty}\left(1-\frac{2}{\pi n}\right) \frac{\tilde{a}_{n}^{p}}{n}\right\}^{\frac{1}{p}}\left\{\sum_{n=1}^{\infty} \frac{\tilde{b}_{n}^{q}}{n}\right\}^{\frac{1}{q}} .
$$


Then by (3.1) and (3.6), it follows that

$$
\frac{1}{2} B\left(\frac{p-\varepsilon}{2 p}, \frac{p+\varepsilon}{2 p}\right) \sum_{n=1}^{\infty} \frac{1}{n^{1+\varepsilon}}>k(1-o(1))^{\frac{1}{p}} \sum_{n=1}^{\infty} \frac{1}{n^{1+\varepsilon}},
$$

and then $\frac{\pi}{2} \geq k\left(\varepsilon \rightarrow 0^{+}\right)$. This contradicts the fact that $k>\frac{\pi}{2}$. Hence the constant factor $\frac{\pi}{2}$ in (3.2) is the best possible. The theorem is proved.

Theorem 3.3 If $0<p<1, \frac{1}{p}+\frac{1}{q}=1, a_{n} \geq 0,0<\sum_{n=1}^{\infty} \frac{a_{n}^{p}}{n}<\infty$, then

$$
\sum_{n=1}^{\infty} n^{p-1}\left(\sum_{m=1}^{\infty} \frac{a_{m}}{m^{2}+n^{2}}\right)^{p}>\left(\frac{\pi}{2}\right)^{p} \sum_{n=1}^{\infty}\left(1-\frac{2}{\pi n}\right) \frac{a_{n}^{p}}{n},
$$

where the constant factor $\left(\frac{\pi}{2}\right)^{p}$ is the best possible.

Proof By the reverse of Hölder's inequality and (3.4), one has

$$
\begin{aligned}
& \left(\sum_{m=1}^{\infty} \frac{a_{m}}{m^{2}+n^{2}}\right)^{p}=\left\{\sum_{m=1}^{\infty}\left[\frac{a_{m}}{\left(m^{2}+n^{2}\right)^{\frac{1}{p}}}\right]\left[\frac{1}{\left(m^{2}+n^{2}\right)^{\frac{1}{q}}}\right]\right\}^{p} \\
\geq & \left\{\sum_{m=1}^{\infty} \frac{a_{m}^{p}}{m^{2}+n^{2}}\right\}\left\{\frac{\omega(n)}{n}\right\}^{p-1}>\left(\frac{\pi}{2 n}\right)^{p-1} \sum_{m=1}^{\infty} \frac{a_{m}^{p}}{m^{2}+n^{2}} .
\end{aligned}
$$

Hence we have

$$
\begin{aligned}
& \sum_{n=1}^{\infty} n^{p-1}\left(\sum_{m=1}^{\infty} \frac{a_{m}}{m^{2}+n^{2}}\right)^{p}>\left(\frac{\pi}{2}\right)^{p-1} \sum_{n=1}^{\infty} \sum_{m=1}^{\infty} \frac{a_{m}^{p}}{m^{2}+n^{2}} . \\
= & \left(\frac{\pi}{2}\right)^{p-1} \sum_{m=1}^{\infty} \sum_{n=1}^{\infty} \frac{1}{m^{2}+n^{2}} a_{m}^{p}=\left(\frac{\pi}{2}\right)^{p-1} \sum_{m=1}^{\infty} \omega(m) \frac{a_{m}^{p}}{m} .
\end{aligned}
$$

By (3.5), we have (3.8).

By the reverse of Hölder's inequality, one has

$$
\begin{aligned}
& \sum_{n=1}^{\infty} \sum_{m=1}^{\infty} \frac{a_{m} b_{n}}{m^{2}+n^{2}}=\sum_{n=1}^{\infty}\left[n^{1 / q} \sum_{m=1}^{\infty} \frac{a_{m}}{m^{2}+n^{2}}\right]\left[n^{-1 / q} b_{n}\right] \\
\geq & \left\{\sum_{n=1}^{\infty} n^{p-1}\left(\sum_{m=1}^{\infty} \frac{a_{m}}{m^{2}+n^{2}}\right)^{p}\right\}^{1 / p}\left\{\sum_{n=1}^{\infty} \frac{b_{n}^{q}}{n}\right\}^{1 / q} .
\end{aligned}
$$

If the constant factor in (3.8) is not the best possible, one can get a contradiction that the constant factor in (3.2) is not the best possible by using (3.9). Hence the constant factor in (3.8) is the best possible. Thus we complete the proof of the theorem.

Remark 3.4 Following (2.6) in $\lambda=2$ and (3.2), one can get a two-sides inequality as:

$$
\left\{\sum_{n=1}^{\infty}\left(1-\frac{2}{\pi n}\right) \frac{a_{n}^{p}}{n}\right\}^{\frac{1}{p}}\left\{\sum_{n=1}^{\infty} \frac{b_{n}^{q}}{n}\right\}^{\frac{1}{q}}<\frac{2}{\pi} \sum_{n=1}^{\infty} \sum_{m=1}^{\infty} \frac{a_{m} b_{n}}{m^{2}+n^{2}}<\left\{\sum_{n=1}^{\infty} \frac{a_{n}^{r}}{n}\right\}^{\frac{1}{r}}\left\{\sum_{n=1}^{\infty} \frac{b_{n}^{s}}{n}\right\}^{\frac{1}{s}},
$$

where $0<p<1, \frac{1}{p}+\frac{1}{q}=1$ and $r>1, \frac{1}{r}+\frac{1}{s}=1$. 


\section{References}

[1] G. H. Hardy, J. E. Littlewood and G. Polya, Inequalities, London, Cambridge Univ. Press, 1952.

[2] D. S. Mitrinovic, J. E. Pecaric and A. M. Fink, Inequalities Involving Functions and Their Integrals and Derivatives, Boston, Kluwer Academic Publishers, 1991.

[3] Bicheng Yang and Mingzhe Gao, On a best value of Hardy-Hilbert's inequality, Advances in Mathematics, 26,2(1997), 159-164.

[4] Mingzhe Gao and Bicheng Yang, On extended Hilbert's inequality, Proceedings of the American Mathematical Society, 126,3(1998), 751-759.

[5] Bicheng Yang, On generalizations of Hardy-Hilbert's inequality and their equivalent form, Journal of Mathematics, 24,1(2004), 24-30.

[6] Bicheng Yang, On an extension of Hardy-Hilbert's inequality , Chinese Annals of Mathematics, 23A,2(2002), 247-254.

[7] Bicheng Yang and T. M. Rassias, On the way of weight coefficient and research for the Hilbert-type inequalities, Mathematical Inequality and Applications, 6,4(2003), 625-658.

[8] Chang jian Zhao, On inverses of disperse and continuous Pachpatte's inequalities, Acta Mathematica Sinica, 46,6(2004), 1111-1116.

[9] Wang Zhuxi, Gua Dunren, An Introduction to Special Functions, Beijing, Since Press, 1979.

Research supported by Natural Science Foundation of Guangdong Institutions of Higher Learning, College and University (China, No.0177), and Appropriative Research Fund for Professors and Doctors, Guangdong Institute of Education.

Received: April 15, 2006 